\title{
Sequence Number Alteration by Logical Transformation (SALT): A Novel Method for Defending Session Hijacking Attack in Mobile Ad hoc Network
}

\author{
N. Nishanth and S. Suresh Babu
}

\begin{abstract}
TCP attacks are the major problem faced by Mobile Ad hoc Networks (MANETs) due to its limited network and host resources. Although the session hijack attack is not in the forefront of everyone's mind, it still remains a commonly used attack in MANET. Session hijack attacks are defined as taking over an active TCP/IP communication session without their permission or knowledge. When implemented successfully, attackers assume the identity of the compromised user, enjoying the same access to resources as the compromised user. Network protocols like FTP, Telnet, and rlogin are especially attractive to the attacker, because of the session oriented nature of their connections, and lack of implementation of any security during logon, authentication, or data transmission. This paper introduces a novel method for defending session hijacking attack in MANET: Sequence number Alteration by Logical Transformation (SALT). Based on the comprehensive evaluation based on experiments, it is showed that SALT can successfully defend session hijacking attack under diverse mobile multi-hop network environment with low communication, computation, and memory overhead.
\end{abstract}

Index Terms - TCP attacks, mobile Ad hoc networks, session hijacking, sequence number, logical transformation.

\section{INTRODUCTION}

The vision of Ubiquitous computing has stimulated much interest in mobile ad hoc networking (MANET) technology. It can turn the dream of getting connected "anywhere at anytime" into reality. In comparison with fixed wireless network, there is no master slave relationship that exists in the MANET. All network activities, such as discovering the topology and delivering data packets, have to be executed by the nodes themselves, either individually or collectively.

MANETs are widely deployed in military battlefield, emergency services like search and rescue mission, cooperative mobile communication etc. Nowadays, they are also widely used in hotels, superstore, offices and airport because they allow easy collaboration and efficient communication on the fly without the need for costly network infrastructure. The unique characteristics of MANETs present a new set of nontrivial challenges to security design. These challenges include open network architecture, shared wireless medium, stringent resource constraints, and highly dynamic network topology [1], [2].

Manuscript received December 9, 2013; revised April 29, 2014

The authors are with the Department of ECE, TKMCE, Kollam, Kerala, India (e-mail: nishtkm@gmail.com,drssbtkm@gmail.com).

\section{A. Attacks in MANET}

The security attacks in MANET can be divided into two groups: Passive attacks and Active attacks. Passive attacks typically involve only eavesdropping of data while active attacks could range from deleting messages, injecting erroneous messages, impersonate a node, etc thus violating the security requirements like availability, integrity, authentication and non-repudiation [3]. In this work, a solution for defending an important attack on Transmission Control Protocol (TCP), namely session hijacking attack in MANET. Major attacks possible in TCP are SYN flooding based Denial of Service (DoS) attack, Session hijacking, Distributed DoS attack and TCP ACK storm.

1) SYN flooding based DoS attack: It exploits the weakness in TCP specifications. In this attack, an attacker sends a large number of spoofed SYN packets to the victim server. Since the SYN request is spoofed, the victim server never receives the final ACK packet from the client to complete the 3-way handshake. Since the backlog queue of victim server is of finite size, flooding of spoofed SYN requests can easily exhaust the victim server's backlog queue, causing all of new incoming legitimate SYN request to be dropped [4].

2) Distributed denial of Service (DDoS) attack: The DoS attack outlined above involve the use of a single client to launch an attack on a system or service. DDoS attacks use the same basic attack methodologies with the exception that the attacks are initiated from multiple client systems (zombies) that are anonymously guided by the attacker. DDoS is also aimed at exhausting the victim network resources such as bandwidth, computing power, etc.

3) TCP ACK Storm: Attacker launches this attack after hijacking a session between two nodes. Now, the attacker sends a TCP packet with an incorrect sequence number to the node. The node now replies with an ACK packet containing the expected sequence number and the originating system sends another packet with the incorrect sequence number. This exchange of ACK packets continues indefinitely back and forth and thus creates a TCP ACK storm [5].

4) Session Hijacking: Session hijack attacks are defined as taking over an active TCP/IP communication session without their permission or knowledge. When implemented successfully, attackers assume the identity of the compromised user, enjoying the same access to resources as the compromised user. Network protocols like FTP, Telnet, and rlogin are especially attractive to the attacker, because of the session oriented nature of 
their connections and lack if implementation of any security during logon, authentication, or data transmission. In fact, data sent using these protocols are sent in clear text which can be easily be viewed by anyone monitoring the network. There are three different types of session hijack attacks; active, passive, and hybrid. The active attack is when the attacker hijacks a session on the network. The attacker will silence one of the machines, usually the client computer, and take over the client's position in the communication exchange between the workstation and the server. The active attack also allows the attacker to issue commands on the network making it possible to create new user accounts on the network, which can later be used to gain access to the network without having to perform the session hijack attack. Passive session hijack attacks are similar to the active attack, but rather than removing the user from the communication session, the attacker monitors the traffic between the workstation and server. The primary motivation for the passive attack is it provides the attacker with the ability to monitor network traffic and potentially discover valuable data or passwords. Hybrid attack is a combination of the active and passive attacks, which allow the attacker to listen to network traffic until something of interest is found. The attacker can then modify the attack by removing the workstation computer from the session, and assuming their identity [6]. At first, the attacker spoofs the victim's IP address, determines the correct sequence number that is expected by the target by sniffing the traffic exchanged between the nodes which is illustrated in Fig. 1, and then performs a DoS attack on the victim.
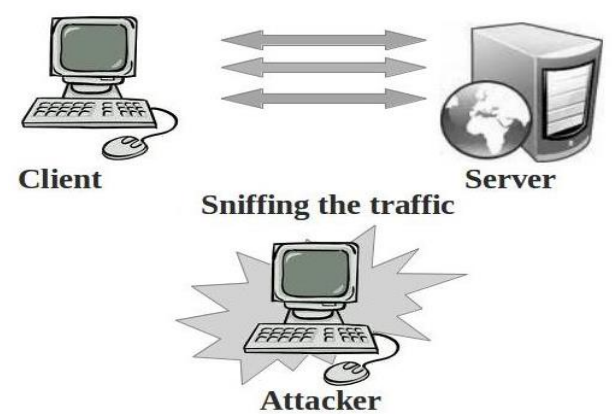

Fig. 1. Attacker passively sniffing the traffic.

As a result, the target system becomes unavailable for some time. Thus the attacker impersonates the victim node continues the session with the target which is illustrated in Fig. 2.

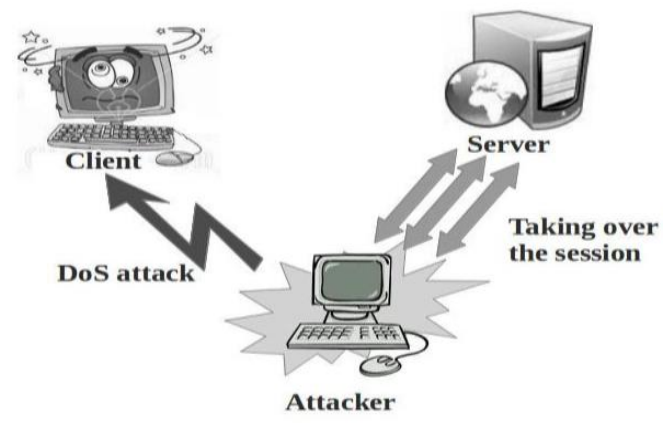

Fig. 2. Attacker taking over the session.
In summary, SYN flooding attack occurs during the time of connection establishment. Attacker sends a large number of SYN packets to the server and floods the resources available for data transmission thereby preventing the legitimate user from making connection. Session hijacking occurs after TCP connection is established. Through the observation of packets, attacker gets knowledge about the sequence number. After getting the knowledge of sequence number, attacker will start sending packets with captured sequence number and hence continue the session with server node.

\section{B. Proposed Idea}

In this paper, a novel method is proposed for defending the session hijacking attack in the network. Session hijacking can be avoided by varying the sequence number and acknowledgment number in a manner which is known only for the communicating nodes and is unknown for the attacker by sniffing the packets.

\section{Organization of Paper}

In Section II, some of the related works on session hijacking is reported. In Section III and Section IV, performance evaluation of the proposed work is reported. Section V summarizes the results.

\section{RELATED WORKS ON DEFENDING SESSION HIJACKING ATTACK}

Despite the wide popularity of MANETs, research into session hijacking attack is limited. Most work concentrates on defending other transport layer attacks like SYN flooding based DoS attack and DDoS attack. Significantly, there are few techniques available for detecting session hijacking attacks that are reliable and effective. Session hijacking attacks, which are usually executed as a combination of identity spoofing and denial of service attacks, cannot be detected very reliably by current systems. An attacker wishing to disrupt a wireless network has a wide variety of tools available to them. Many of these tools rely on using a faked MAC address, masquerading as an authorized client. Using these tools, an attacker can launch denial of service attacks and session hijacking attack. An attacker may choose to alter their MAC address for several reasons, including obfuscating their presence on a network, to bypass access control lists, or to impersonate an already authenticated user. For detecting session hijacking and spoofing attacks, the only mechanism used is based on detecting abnormal jump in sequence numbering of IEEE 802.11 frames or checking if the MAC address belongs to a well known manufacturer. But neither is a very good indicator of a session hijacking or spoofing attack as the sequence numbers range only from 0 to 4096 and gets reset every time the station restarts and the MAC addresses can easily be masqueraded. The sequence numbers are predictable and are not cryptographically protected so can easily be eavesdropped.

Existing approaches for detecting session hijacking attacks include the monitoring of MAC frame sequence numbers, with dramatic changes in sequence numbers indicative of an intrusion. Once a session is taken over by the attacker, we cannot identify the session hijacking. Josua Wright et al. 
proposes solution for detecting MAC address spoofing based on sequence number analysis in MAC header in management frames and data frames. Wright proposes to use sequence number gaps as the detection clue [7]. If the gap exceeds a certain threshold, a spoofing alert is raised. This method, however, may raise false alerts in the presence of lost or duplicated frames, which are common in practice. Guo et al. extend this method to use ARP to confirm the current sequence number from the genuine station, thus reducing false positives [8]. Yong Sheng et al also suggested a method for detecting MAC address spoofing using Received Signal Strength (RSS) [9]. RSS is a measure of the energy observed by the physical layer at the antenna of a receiver. The RSS is a measurement that is hard to forge arbitrarily and it is highly correlated to the transmitter's location. Assuming the attacker and the victim are separated by a reasonable distance, RSS can be used to differentiate them to detect MAC spoofing. Every radio transceiver has unique physical characteristics, which lead to unique patterns in the RF signals it transmits. Hall et al. propose to identify a transceiver and thus detect spoofing using transceiver prints [10]. They use a wavelet transform to extract frequency domain features of the transient portion of RF signals, and use fuzzy neural networks to determine whether a given signal matches the profiles or not. Rupinder Gill et al. suggested two techniques that can be used by a Wireless Intrusion Detection System (WIDS) to passively detect session hijacking attacks. The strength of RF signals undergoes some attenuation during transmission after leaving the sender's radio and this signal strength deterioration is governed by a variety of factors like RF interferences, distance between communicating nodes, obstacles etc. The distance between the two nodes has the biggest impact on signal fading. However, RF signal strength does not fade in a linear manner; rather it attenuates roughly inversely as the square of the distance between the two nodes [11]. Along with distance, the RSS for a particular node, as observed by the receiver, also depends on various other factors like the WLAN equipment used by both the sender and the receiver nodes, the physical obstacles in between and their surrounding environment. Thus, the adversary will need to be at exactly the same location as the receiver, use exactly the same radio equipment, and receive the radio signal with same level of interference, reflections and refractions to know the exact RSS value as perceived by the receiver. Even if the sender is stationary, RSS values tend to slightly fluctuate and hence prove almost impossible to guess. This prohibits the adversary from using radio equipment (like a high gain directional antenna) to spoof the RSS as perceived by the receiver.

But these methods are infeasible in mobile ad hoc networks. Since, in order to identify session hijacking each node in the network should do frequent and complex computation regarding RSS and should updated regularly which is not practical in resource restricted environments like MANET and wireless sensor networks (WSN).

Hence, it necessitates designing a mechanism to avoid session hijacking which is robust to mobility, untrusted environment and should consume only lower CPU, battery and memory resources. Keeping these factors in mind, this work proposes Sequence number Alteration by Logical
Transformation (SALT).

\section{THE SALT METHOD}

When a node wants to communicate with another node, it should initiate a 3-way handshake. Once the 3-way handshake is completed, the nodes will exchange the data packets. If an attacker is present in the network, he will passively monitor the traffic exchanged between the nodes. Once the attacker gets the information about the sequence number present in the TCP header, it will initiate a DoS attack to the client node. Now, the attacker impersonates the client node and continues the session with the server node. But, the server node is unaware about this impersonation. The SALT method is an efficient solution to prevent session hijacking by changing the sequence number by a logical function which is known only for the legitimate nodes present in the network so that the attacker is unaware about the exact sequence number used in the session. By sniffing the traffic, the attacker will get only the transformed sequence number which is completely different from the original sequence number. The sequence number is altered using a function $F$, and then forward to the next node.

$$
\hat{S}=F(S)
$$

where $\hat{S}$ is the transformed sequence number and $S$ is the original sequence number and $F$ is the logical transformation which is known only to the legitimate nodes in the network. In order to find the exact sequence number by the server node, it will perform an inverse transformation such that it will get the original sequence number and continue the communication.

$$
S=F^{*}(\hat{S})
$$

where $F^{*}$ is the inverse transformation which is known only to the legitimate nodes in the network.

\section{A. The Network Model}

Consider a network with 10 nodes and for the experimental purpose, one attacker is deployed near to the two nodes as shown in the Fig. 3. For the illustration purpose, consider an example of communication between a client node and a server node in the network. Here, $F$ is logical transformation rotate right by 1 and $F^{*}$ is the logical transformation rotate left by 1 .

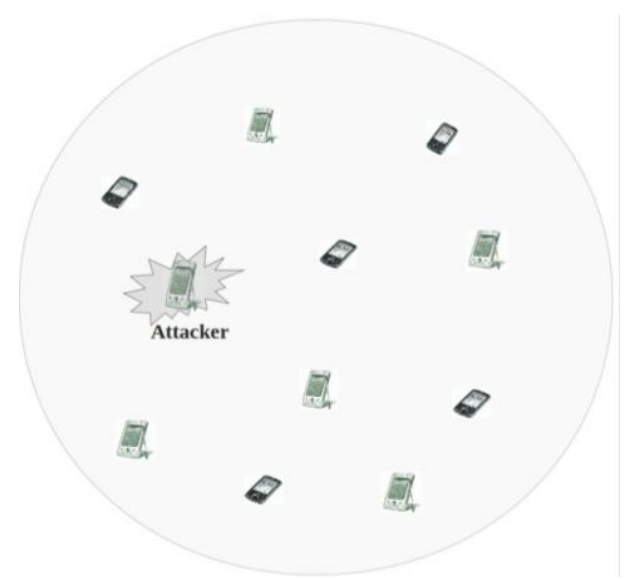

Fig. 3. Network model. 
Assume that initial sequence number chosen by the client node be 1 and convey this information to the server node during 3-way handshake. The server node chooses its sequence number as 10 and it will increment the sequence number from the client node and put it in the acknowledgement number field which is illustrated in the Fig. 4.

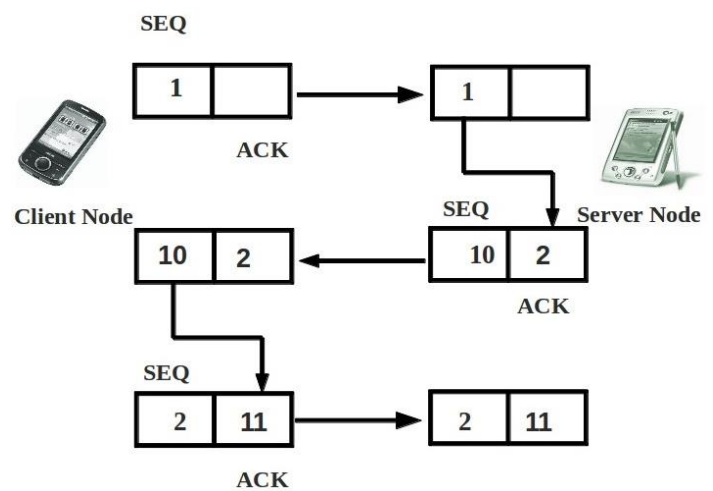

Fig. 4. Sequence number before logical transformation.

By introducing the SALT method, the sequence number undergoes a logical transformation before sending it to the server node. The server node will perform the inverse transformation in order to get back the original number which is illustrated in Fig. 5.
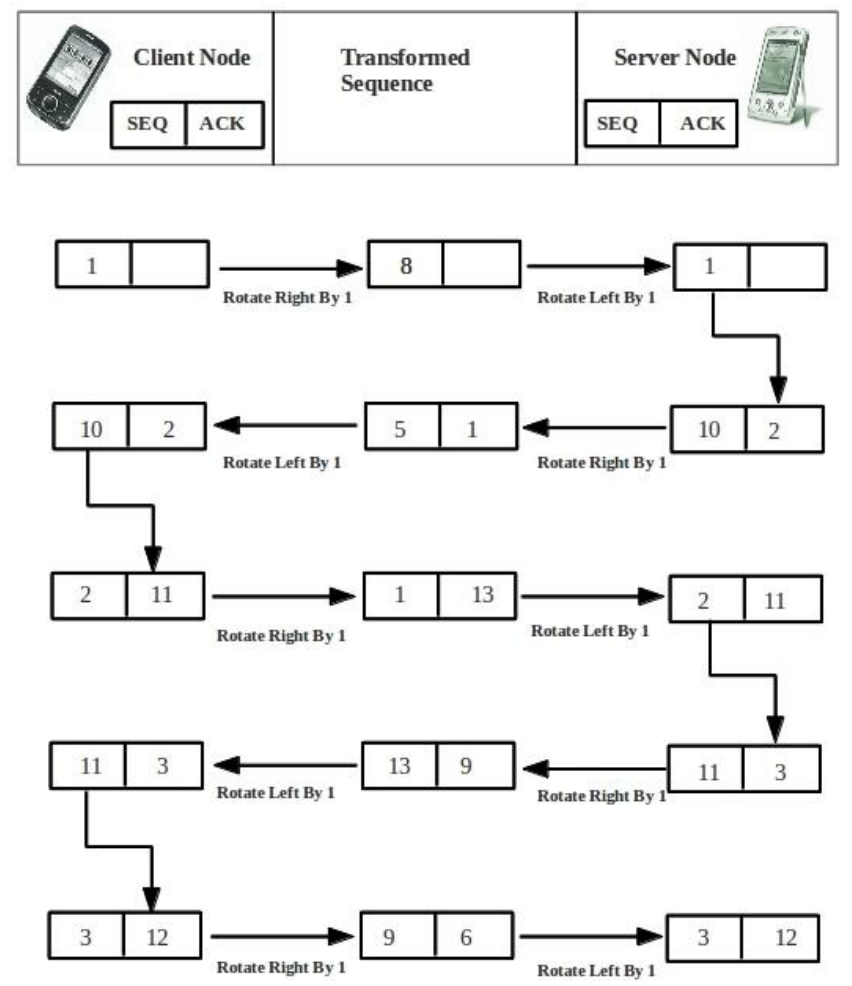

Fig. 5. Procedure for SALT method.

By using SALT method, the client node rotate right the sequence number by 1 . So we get 8 (1000) as new sequence number. It will reach at the server node. Then it will be converted to $1(0001)$ by rotate left by 1 operation. Then 1 will be incremented to 2(0010) and is used as the next ACK number. The server node chooses its sequence number as 10(1010). Then this operation will continues and is illustrated in the Fig. 6.

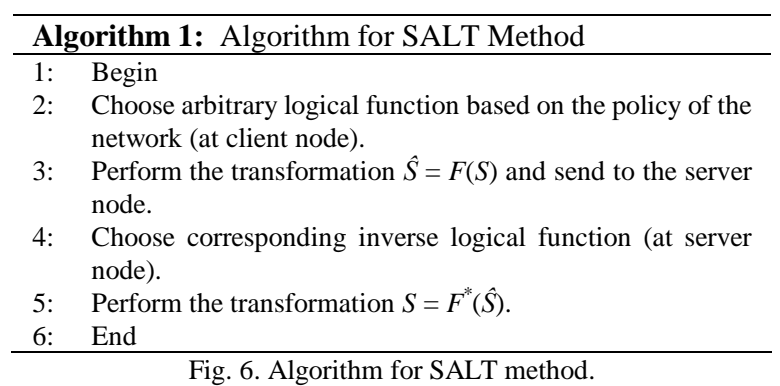

The above illustration is done by considering a 4-bit sequence number and acknowledgement number. For an attacker unaware of the logical function used for transformation, feels that variation of sequence number and acknowledgement number is completely random in nature.

The success of the SALT method depends on the logical function, $\mathrm{F}$ used for the transformation of sequence number and acknowledge number. The requirement of the algorithm is that, by observing sufficient number of packets, the attacker may not be able to predict the logical function, F used for the transformation. Since, the sequence number and acknowledgement number in TCP protocol is 32-bit, it is very difficult for an attacker to identify the logical function used for transformation.

\section{EXPERIMENTAL RESULTS}

For studying the statistics for normal traffic, experiments are conducted in our lab for two months. Even though tcpdump [12] can be used for the analysis of TCP traffic in the network, a packet capture program is written in $\mathrm{C}$ language for our convenience. The screenshot of the experiment after implementing the SALT method is as shown in Fig. 7.

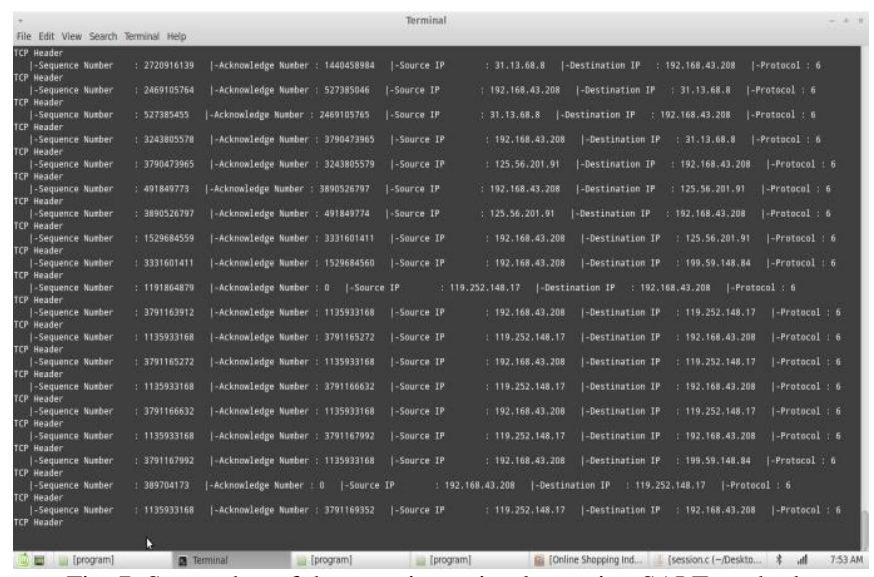

Fig. 7. Screenshot of the experiment implementing SALT method.

Separate C program is written for predicting the logical function from the information present in the captured packet. The algorithm can be improved by changing the logical function used for transformation at regular interval. The choice of the logical function is known for the legitimate nodes present in the network. For evaluation of the performance of our method, we used the parameter, Attack Success Rate (ASR) which is defined as the ratio of number of times an attacker successfully launch session hijacking attack to the total number of attack attempt. A typical plot of ASR with time is as shown in the Fig. 8. 


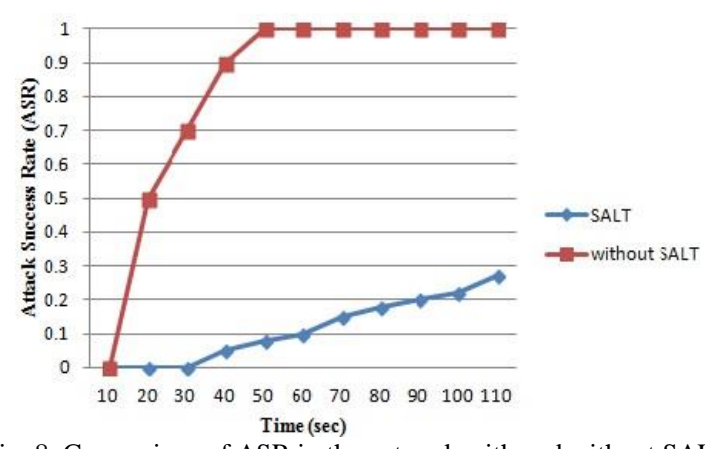

Fig. 8. Comparison of ASR in the network with and without SALT algorithm.

A comparison of ASR in the network has been carried out as the time evolves. It is seen that ASR is very much less in the network having SALT algorithm. It could be see that as time evolves, the proposed algorithm is getting prone to session hijacking attack since the attacker may identify the logical function from the analysis of large number of packets. A comparison also carried out for the computational overhead (in clock cycles/sec) for the network with the number of nodes which is illustrated in Fig. 9.

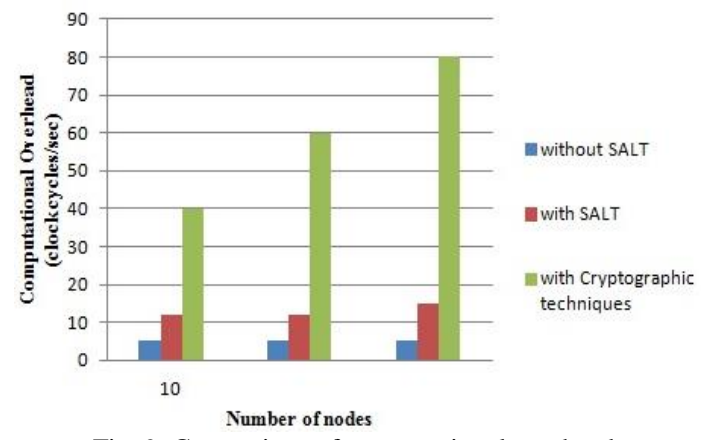

Fig. 9. Comparison of computational overhead.

\section{CONCLUSION}

In this paper, a novel scheme, SALT is proposed for avoiding session hijacking attack in MANET environment. Major advantages of SALT are lower communication and computational complexity associated with avoiding the attack These facts were proved with the comprehensive evaluation based on simulations. As seen from the analysis, the SALT algorithm is prone to session hijacking attack as the time evolves since the attacker may be possible to identify the logical function from the analysis of large number of packets.

\section{REFERENCES}

[1] N. Nishanth and P. Venkataraman, "Mobile agent based TCP attacker identification in MANET using the traffic history (MAITH)," in Proc. 13th IEEE Int. Conf. on Communication Technology (ICCT), 2011, pp. 1130-1134.
[2] W. Li and A. Joshi, "Security issues in mobile ad hoc networks- A survey," The 17th White House Papers Graduate Research In Informatics at Sussex, 2004, pp. 1-23.

[3] K. Pelechrinis, M. Iliofotou, and S. V. Krishnamurthy, "Denial of service attacks in wireless networks: The case of Jammers," IEEE Communications Surveys and Tutorials, vol. 13, issue 2, 2011.

[4] H. Wang, D. Zhang, and K. G. Shin, "Detecting SYN flooding attacks," in Proc. IEEE INFOCOM, 2002, pp. 1530-1539.

[5] K. Biswas and M. L. Ali, "Security threats in mobile ad hoc network," School of Engineering, Blekinge Institute of Technology, Sweden.

[6] P. Jess, "Session hijacking in windows network," SANS Institute InfoSec Reading room, 2006.

[7] J. Wright. (2003). Detecting wireless LAN MAC address spoofing. Technical Document. [Online]. Available: http://home.jwu.edu/jwright

[8] F. Guo and T. Chiueh, "Sequence number-based MAC address spoof detection," in Proc. the 8th International Symposium on Recent Advances in Intrusion Detection, Seattle, WA, USA, Sept. 2005.

[9] Y. Sheng, K. Tan, G. Chen, D. Kotz, and A. Campbell, Detecting 802.11 MAC Layer Spoofing Using Received Signal Strength.

[10] J. Hall, M. Bareau, and E. Kranakis, "Using transceiverprints for anomaly based intrusion detection," in Proc. $3^{\text {rd }}$ IASTED, CIIT, Nov. 2004, p. 2224.

[11] R. Gill, J. Smith, M. Looi, and A. Clark, "Passive techniques for detecting session hijacking attacks in IEEE 802.11 wireless networks," in Proc. AusCERT Asia Pacific Information Technology Security Conference, 2005.

[12] Tcpdump public repository. [Online]. Available: http://www.tcpdump.org

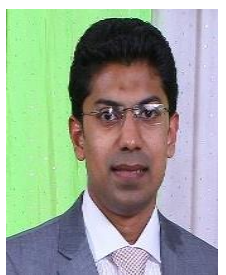

N. Nishanth was born in Kerala, India on January 5 , 1982. He completed his B.tech in electronics \& communication engineering from College of Engineering Trivandrum, Kerala in 2003. He took his M.E (Telecom) degree from Indian Institute of Science (IISc), Bangalore, India in the year 2011. His major area of interest is computer networks and its security, security for MANET etc.

$\mathrm{He}$ is currently working as an assistant professor in Department of ECE, T K M College of Engineering, Kollam, Kerala. He has around 10 years of teaching experience.

Prof. Nishanth is an active member of IEEE and currently holding the position of Secretary of IA/IE/PEL section, Kerala Chapter.

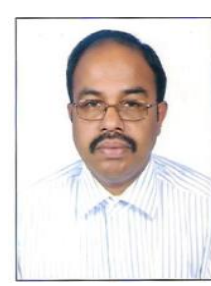

S. Suresh Babu received his B.Tech degree in electronics \& communication engineering from T K M College of Engineering, University of Kerala, in 1982. He received the $M$. Tech degree in computer science \& technology from IIT Roorkee in 1988 and $\mathrm{PhD}$ degree in IT enabled services from PSG College of Technology in 1997. From 1997, he had served as principal at various engineering colleges in Kerala including T K M College of Engineering. His research interests include IT enabled services, digital image processing, and Ad hoc networks.

Dr. S. Suresh Babu is a member of both Board of Studies \& Faculty of Engineering of CUSAT. He is also a member of PG Board of Studies (Engineering \& Technology) in the University of Kerala. Currently, he is working as a professor and the head of the Department of Electronics \& Communication Engineering, T K M College of Engineering, Kollam. 